\title{
Design and Realization of Foreign Language Learning System based on Mobile Internet Platform
}

\author{
Chu Weili
}

\author{
Qingdao Huanghai University
}

\section{Keywords: Mobile internet, Foreign language learning system, Podcast}

\begin{abstract}
The paper aims to develop a set of mobile Internet platform system for the foreign language teaching. The background and purpose of the system design are introduced briefly, the relatively detailed demand analysis is made for the system, including clearing the problem definition and feasibility analysis. The establishment of system structure model is completed in the profile design phases, the database and interface are designed, and each module to be realized for the system is designed in details according to the system structure.
\end{abstract}

\section{Introduction}

Lessons in the school, companies providing the paid English training and online autonomous learning are the main means to learn English currently, while online learning has become the most convenient English learning channel obtaining the most information widely used by the learners. We can naturally think that it is obvious for the handheld device to surpass the portable computer along with the advent of the era of mobile Internet, and people hope to learn English via the mobile phone inevitably[1]. It is more flexible and portable for the mobile phone when compared with other above learning means, it has little restrictions on the time and location, and better experience can be brought for the users[2]. Therefore, it is necessary to develop a practical English learning platform under the currently prevailing Android system.

An application of English Podcast is realized in Android platform in the paper. Podcast is a prevailing broadcasting form via the network to get the acoustic signal information with the main feature of autonomous broadcasting, which is also the fundamental difference from the traditional broadcasting[3]. It is passive to hear the traditional broadcasting, while we need to locate to a particular frequency and listen at the specific period of time in the real-time listening of the program we want with a relatively low flexibility[4]. Our favorite program can be customized according to personal preference in the new broadcasting form of Podcast.

The background and purpose of the system design are introduced briefly, the relatively detailed demand analysis is made for the system, including clearing the problem definition and feasibility analysis. The establishment of system structure model is completed in the profile design phases, the database and interface are designed, and each module to be realized for the system is designed in details according to the system structure[5]. The code realization is conducted for each module in the realization part, and the problems needing to be considered in the coding are combed. Finally, the black-box testing way is used mainly to design the test case for each module. 


\section{System analysis and design}

The basic objective of the system analysis is to determine how the system completes the scheduled task in a relatively abstract and generalized way. During the period, the physical configuration program of the system is mainly completed, thus the structural design of each program in the system is realized. Two sub-phases are included in the profile design phase: system design and software structure design. The system design is to set up the reasonable realization program from the data flow chart obtained via the demand analysis; and the software structure design is mainly determining the several modules constituting the software, the correlation and dynamic calling relationship among the several modules.

The advantage of profile design before the detailed design is that the developer can analyze the system design program and software structure from the relatively abstract degree with the minimal cost at the global height, thus developing the high-quality software system with low cost.

The definition and division of module shall meet the design principle of high cohesion and low coupling when designing the software structure. The structural hierarchy of the software can be seen in Figure 1.

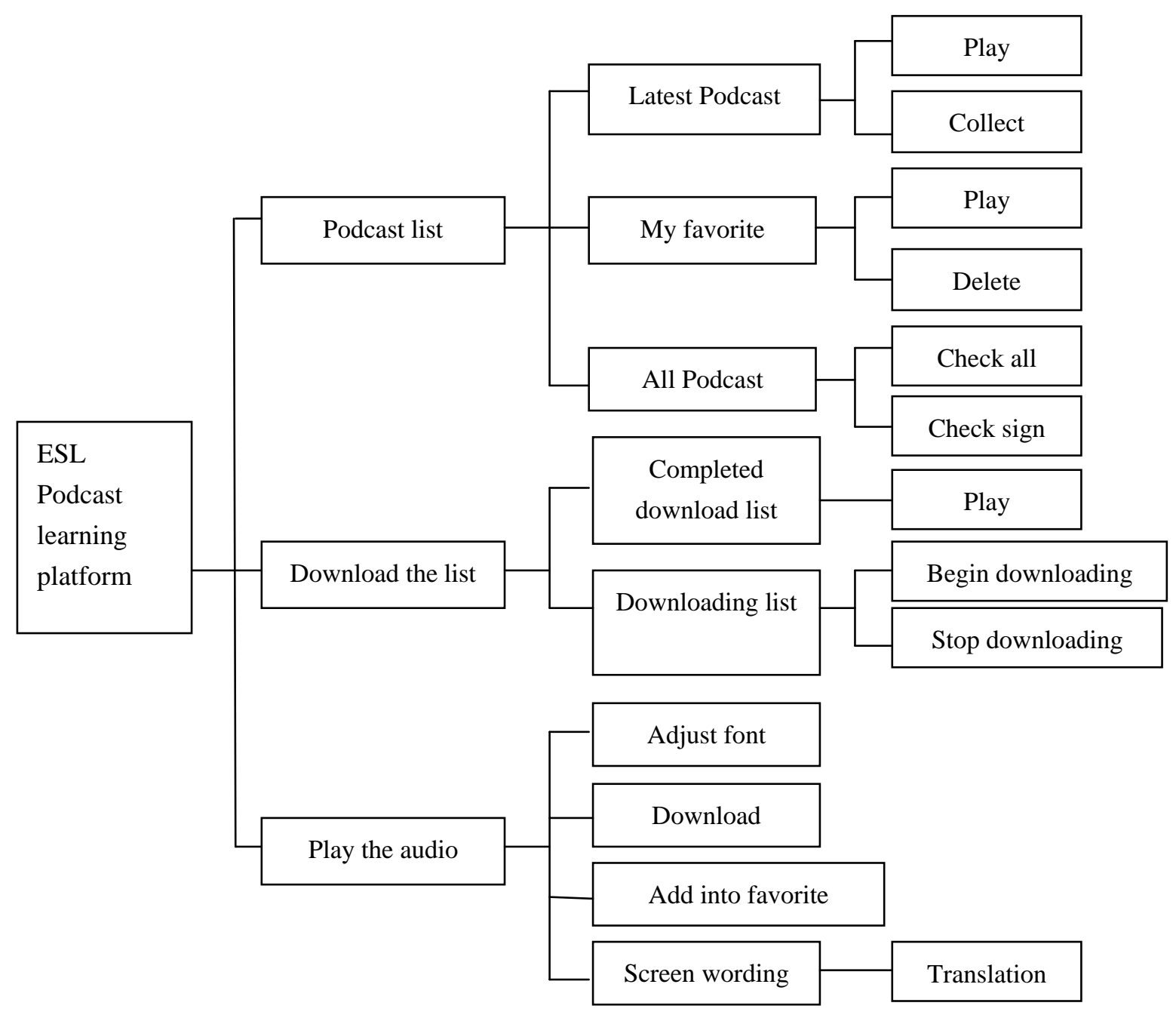

Figure 1 Structural hierarchy of the system 


\section{Database design}

Nowadays, people's field of version is broader and broader and the data amount is increased sharply along with the rapid development of science and technology, and how to save and manage the great and complex data to facilitate and fully use the valuable information resources are to be solved for the database design. The database is stored in the computer for a long time and organized and shared huge data favorites, the data in the database is equipped with the smaller redundancy, higher data independence and expansibility. Using the database storage and interactive data is more efficient than $\mathrm{I} / \mathrm{O}$ interaction for the development carrier of mobile phone with relatively strict internal storage non-matching requirement. Therefore, designing the database rationally and using the data resource efficiently must be considered and processed in the development of handheld equipment application.

The objective of database design is to provide the operating environment of information infrastructure and high efficiency for users and various application systems.

The efficiency of operating environment mainly includes the following three points:

1. Access efficiency of database data.

2. Utilization of database storage space.

3. Efficiency of operating management of database system.

The table structure involved in the system is as follows:

(1) User favorite table (tb_Favorite), ID (favorld) of favorite podcast is primary key to save the user favorite information as shown in Table 1. The title of favorite podcast is saved in the table, the text profile is displayed in the favorite list, and the audio and original text are used to open the complete text and play the audio.

Table 1 Users' favorites sheet

\begin{tabular}{|c|c|c|c|c|}
\hline Field name & Meaning & Data type & Null & Field note \\
\hline favored & $\begin{array}{c}\text { Favorite podcast } \\
\text { ID }\end{array}$ & Int & Main key \\
\hline UserID & User ID & Var & Y & (Not used) \\
\hline Title & Title of text & Var & Y & Y \\
\hline Brief & Content of text & Var & Y & Point to network \\
audioPath & $\begin{array}{c}\text { Network audio } \\
\text { path }\end{array}$ & Var & Y & $\begin{array}{c}\text { Point to network } \\
\text { path }\end{array}$ \\
\hline audioText & $\begin{array}{c}\text { Network audio } \\
\text { text }\end{array}$ & Var & & \\
\hline
\end{tabular}

(2) User download data table (tb_Downloaddata), downloading the text ID (DLID) is the main key to save the user's downloaded data information as shown in Table 2. The data in the table is used to display the download list interface, the title and content are used to display the list, and the local path is used to play the audio. 
Table 2 Users to download data sheets

\begin{tabular}{|c|c|c|c|c|}
\hline Field name & Meaning & Data type & Null & Field note \\
\hline DLID & $\begin{array}{c}\text { Download text } \\
\text { code }\end{array}$ & Int & Main key \\
\hline dTitle & $\begin{array}{c}\text { Download text } \\
\text { title }\end{array}$ & Var & $\mathrm{Y}$ & \\
\hline dAudioText & $\begin{array}{c}\text { Download text } \\
\text { content }\end{array}$ & Var & $\mathrm{Y}$ & Point to network \\
path
\end{tabular}

\section{Interface design}

The most important part in the human-computer interaction process is the interface design. The interface can be divided into two levels of feelings (visual, tactile, auditory, etc.) and emotions. The design of user interface is a vital part of screen product. Three major principles for the design of user interface include: place the interface in the control of user; reduce user's memory burden; and maintain the consistency of interface. The factors of interface aesthetics, consistency and integrity of interfaces with different resolution screens, touch screen interface design and others shall also be considered when designing the application interface of the mobile phone. The main interface of ESL podcast is designed and developed based on the above principles.

\section{System application}

The system is a mobile application of ESL podcast realized under Android platform with the main purpose of enabling users to listen to the podcast in ESL blog website via mobile device. The application is attractive for the users needing to listen to English podcast due to the concise and clear design interface, simple operation and relatively perfect function.

The procedure amount of the whole system is not large, and the size of interpreted apk document is about $1.2 \mathrm{M}$. The network connection and page skipping are mainly considered for the operating efficiency of the program. It takes about 3-5s to connect the network, get the website content and display in the screen, and it is less than 1s from the page skipping to the complete display. However, the execution efficiency of the program is reduced due to some redundant code and excessive database interaction among the codes.

Some mainstream Android software platform websites have been uploaded to the system currently. The downloads are increased gradually via a period of statistics, and that in Android market has reached more than 7000 times. The users hold high evaluations on the system for easier operation and smooth usage.

\section{Conclusion}

The function of the system has been perfect preliminary, it will be optimized and upgraded according to the user's online feedback and the appearance of new demand, and transplanted 
possibly at other versions of Android system with huger gap and developed in other mobile platforms.

\section{References}

[1] Li H, Liu H Y, Liu X G, et al. Resources Sharing Patterns Based on Cloud Computing[J]. Journal of Applied Science and Engineering Innovation, 2016, 3(5): 185-188.

[2] Chun D, Kern R, Smith B. Technology in language use, language teaching, and language learning[J]. The Modern Language Journal, 2016, 100(S1): 64-80.

[3] Wang L, Liu G. The Manpower Planning Model for Optimizing Human Resource Based on Flow Cost[J]. Journal of Applied Science and Engineering Innovation, 2016, 3(6): 237-241.

[4] Burston J. Realizing the potential of mobile phone technology for language learning[J]. IALLT Journal of Language Learning Technologies, 2016, 41(2).

[5] Chang C, Chang C K, Shih J L. Motivational strategies in a mobile inquiry-based language learning setting[J]. System, 2016, 59: 100-115. 